\author{
Events and Collusions: a glossary for the microethnography of videogame play \\ [draft version] \\ Published in Games and Culture, 4(2) April 2009 \\ Seth Giddings, University of the West of England, Bristol \\ seth.giddings@uwe.ac.uk http://www.badnewthings.co.uk
}

Two Microsoft X-Box consoles are linked together in a small terraced house in south Bristol. One-in the front room-is connected to a large television screen, the other-in the dining room - to a data projector pointed at a wall cleared of furniture and pictures. It takes some time to persuade the consoles to speak to one another, to recognize that they should divide a videogame, Halo 2, between them, four players per console, but generate a virtual world in which all eight can interact. Each of the two screens (the television and the wall projection) is split into four subscreens, each subscreen displaying the game's virtual world from the point of view of one player. Through their own window, each player sees the game world from the position of their avatar, a first person viewpoint, from which they can see the avatars of the other players. The players sit together, and their virtual representatives leap and sprint through the game and across the screens, triggering a frenzy of virtual gunfire.

It quickly becomes apparent that my fellow players, despite their claims to incompetence, are much more accomplished than me at this game. Denied the leisurely and tentative exploration of controls, conventions and game world that a single-player version of the game would allow, I am left flailing around. Unable to simultaneously move through the virtual space, identify useful power-ups and ammunition and aim my virtual weapon at my on-screen enemies, my avatar is shot repeatedly, hitting the floor with frustrating regularity and frequency. In each of these deaths my screen point-of-view is spun through 90 degrees as my avatar hits the floor, capturing-before momentary virtual oblivion and respawningthe perpetrator of this temporary downfall, skipping blithely away to engage with more worthy opponents.

I try instead to explore the game world, to take my avatar for a walk. The virtual environment is spectacularly rendered, a beautiful alien landscape. It soon becomes clear, however, that its ostensibly open vistas are illusory-all paths curve gently back into the mayhem. Enticing foothills are in reality, barriers to the inaccessible mountains beyond. This is not a world but a park, a garden landscaped for virtual warfare, an arena. Positioning my avatar behind a rock as far from the explosions and tiny leaping figures in the middle distance as possible, I instead head off-in actual space this time-to the kitchen. Both my physical body and my avatar take a break from this intense and frustrating play.

Drinking a beer and eating crisps I watch my actual opponents now, rather than the screens. In contrast to the intense onscreen activity-leaping fighters, exploding vehicles, wild virtual camera swings - the players are immobile. Occasional unexpected deaths or particularly spectacular moments of action are met with curses or laughter, and the end of a match is marked by the stretching of backs and arms, but in play the players are still, eyes wide and rarely blinking, motion evident only in the micromovements of thumbs on controller buttons and analogue sticks. A phrase from Donna Haraway's, "A Manifesto for Cyborgs" flits into my mind: "our machines are disturbingly lively, and we ourselves frighteningly inert" (Haraway, 1990, p. 194). My own sense of heterogeneous agency in the game (paradoxically highlighted by my ineptitude), of driving my avatar to wild movement, to triggering actions, operating machinery, firing weapons, navigating spaces and buildings, is palpable and intense, yet profoundly at odds with this scene of transfixed bodies and minds. They seem 
not to be initiating the on-screen action or making choices-as conventional celebrations of interactivity might assume-but rather responding to the demands of the machines (both the hardware and software).

The term "cyborg" does not seem too hyperbolic here. On the one hand, the players are temporarily, but intensely, locked in a circuit-a cybernetic feedback loop-in which they, the consoles, controllers, and the game-software are nodes. The linguistic confusion evident in my account of the game above is telling: "I" referring at times to my physical body and sense of self, but at times to my actions in the game. There is a linguistic and experiential blurring of boundaries between human and machines: in the game "l" is at once "myself", "my avatar", and "myself and avatar"; a hybrid human and technological entity.

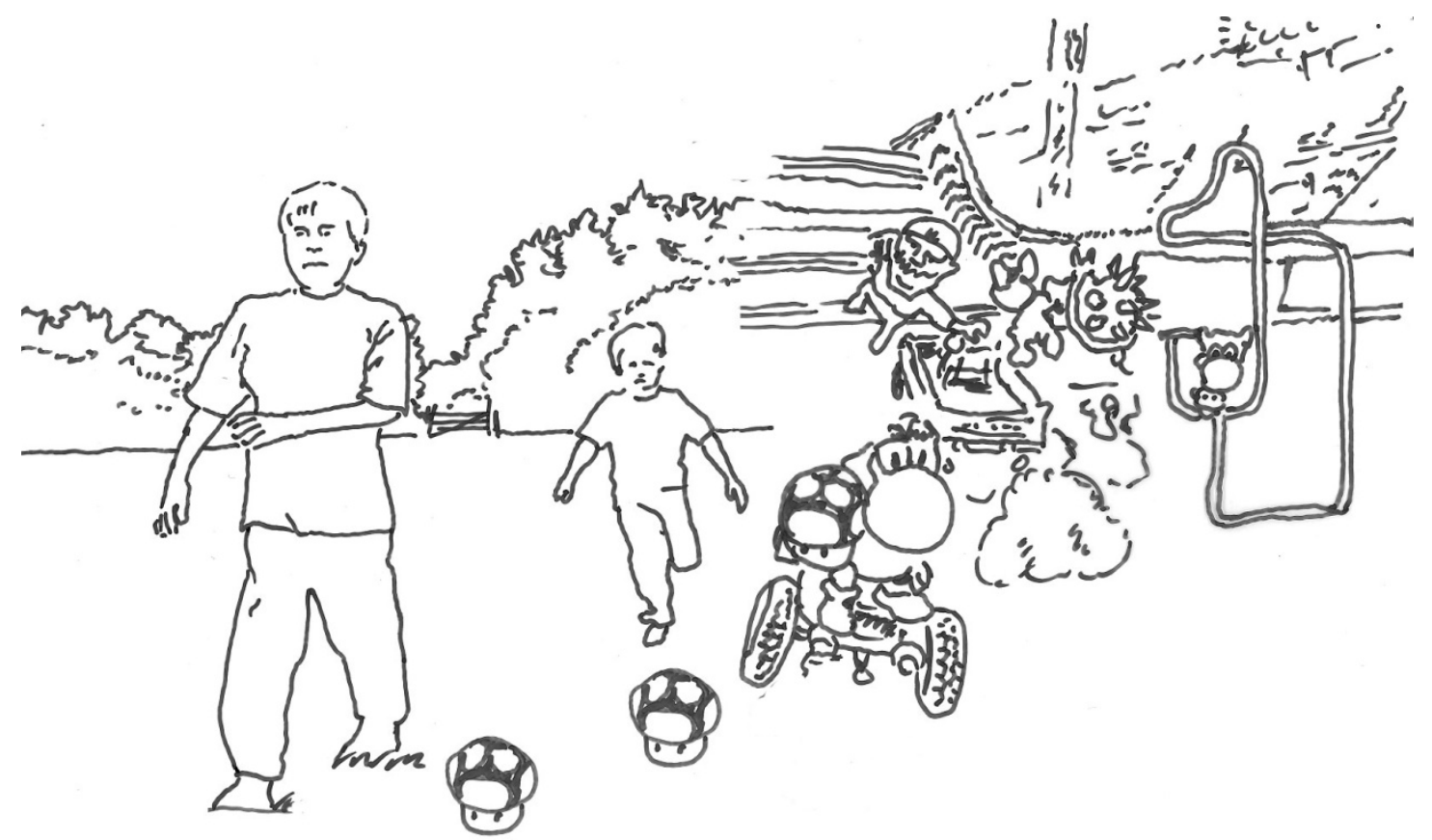

Another, very different, space, but again ludically charged. We are on a family holiday, camping in the Doone Valley on the edge of Exmoor in Dorset. The setting for R. D. Blackmore's 19th-century novel Lorna Doone, the small, enclosed valley is full of references to its literary incarnation. The local heritage and tourist industry has erected signs indicating both contemporary footpaths and sites thought to correspond to places and events in the book. This is then an actual place overlaid or dramatized by the virtual time-space of literature.

On our walk back to the campsite along the river, my wife Penny energizes our flagging children by pointing out mushrooms growing amongst the grass and mentioning Mario Kart. This triggers an explosion of activity in the boys. They sprint across the field, slowing down then accelerating noisily as they reach another patch of mushrooms. Mario Kart: Double Dash!! is a videogame for the Nintendo GameCube and a firm favourite in our house. One convention of this genre of game-an arcade-style racing game-is the inclusion of powerups or items that bestow temporary powers on the car/avatar. Mushrooms, in this particular game, give the car/avatar a brief but often decisive turn of speed. In the Doone Valley, actual fungus becomes analogous with the bright red and white mushrooms in the game, virtual power-ups in an actual game. 


\section{Anxious Objects}

What are the objects of study in gameplay research? Who or what are the agents and what is the nature of the field in which ethnographic fieldwork might be conducted? These two short stories indicate that some understanding and analysis of the forms, aesthetics and conventions of videogames as popular digital media is very useful, as is the acknowledgement and description of the physical and social context in which these forms and conventions are realized, vivified.

But how to articulate these, how to describe, and analyze particular and general moments or modes of gameplay, factoring in both media form and lived experience? How, for instance, to account for the machinations of affect, as virtual worlds are actualized in the very different time-space of outdoor play? And, crucially, what is the nature of the game player in these intimate circuits of twitching thumbs and lively machines?

This article sets out a working glossary of concepts and terms that have arisen from some recent analytical and ethnographic research. Some of these terms are appropriated or repurposed from existing theoretical and ethnographic approaches; a few are new to the field. I will refer to two recent small-scale research projects that make use of video to record and analyze videogame play. I will address the methodological demands of studying the relationships between the virtual and the actual, cyberspace and domestic space, in everyday popular digital culture. I also attempt to identify and theorize both human and nonhuman agents in the moment or event of gameplay: the object of study here is neither human players nor dynamic software but what happens when they come together to generate an event of gameplay.

A methodological requirement for these studies was the breaking down of an entrenched division of epistemological labour in new media research: that between empirical and ethnographic work on the one hand and textual or aesthetic analysis on the other. Videogames are at once computer software with procedural agencies and autonomous operations, computer hardware, and media texts with screen images, sounds, environments, and dramas. I propose then on the one hand (with apologies to Espen Aarseth), a "cybertextual analysis", and on the other, a very small-scale ethnography of videogames, videogame play and videogame players. A synthesis between these analytical and ethnographic methods is needed, given my unwillingness to establish an a priori asymmetry between videogame (as "text"), videogame play (as consumption or practice) and videogame player (as embodied media subject). I adopt the term microethnography (and adapted it as a methodology) to describe this synthetic approach, subsequently suggesting microethology as a more precise term.

\section{Cybertextual Analysis}

Videogames are a significant popular form of screen media, often drawing on films and television for their characters, images, and scenarios. They are also computer software and as such, their algorithmic and procedural operations and structures lie outside the purview of established modes of textual analysis in literary, film, and media studies. New media studies and game studies have begun the project of cybertextual analysis (Aarseth, 1997; Bolter \& Grusin, 1999; Lister, Dovey, Giddings, Kelly, \&Grant, 2003; Manovich, 2001). Espen Aarseth's book, Cybertext: Perspectives on Ergodic Literature for instance, both engages with the distinct internal operations of computer-based media, and emphasizes the role of the 
reader/player in realizing these cybernetic operations in each act of reading/playing (Aarseth, 1997; see also Salen \& Zimmerman, 2003; Giddings \& Kennedy, 2008).

\section{Part(icipant)s}

Aarseth's (1997) diagram of a text-based computer adventure game indicates the dynamic, cybernetic circuits of agency between software and hardware components, between databases and software engines, user input and algorithmic processing of information and responses. His alternative term for these components, part(icipant)s, is a rich one as it emphasizes the active role of these parts, parts that come together to constitute any particular instantiation of the adventure game in play. Although Aarseth does not pursue this, the diagram's refusal to grant any other status to the human part(icipant) is suggestive for rethinking the relationships between, and status of, the human and the technological in videogame play.
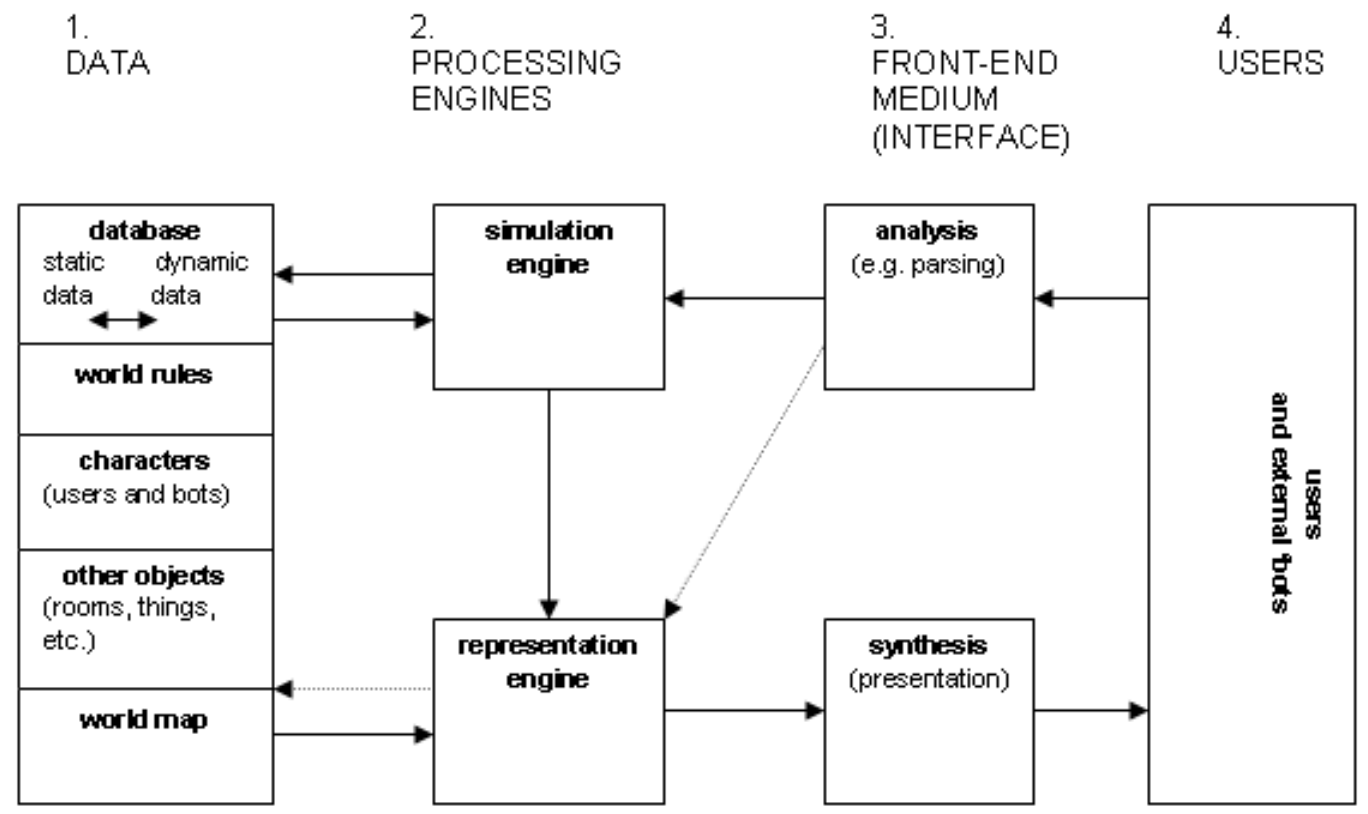

"a generalized conceptualization of the functionality of a typical, but advanced, adventure game" (Aarseth 1997: 103).

Popular computer media then must be conceptualized as both symbolic and material. On the one hand, videogames are toys, popular media, performative events, often characterized by symbolic content derived from established popular screen media. On the other hand, the analysis of videogames as a computer-based medium demands the description of a special category of nonhumans, software entities (in the language of computing, "agents") that act more or less autonomously, or effect emergent behaviour. We should resist conceiving of the videogame as a discrete and "whole" object. The videogame is constituted by software components that effect their own operations and semiautonomous agency within the videogame system. Game worlds and temporalities, modes of presentation, puzzles and combat, engagement with computer-controlled characters, are all constantly configuring the player's experience and responding to the player's responses. 


\section{Microethnography}

I use the term microethnography to describe a nonscientific, improvised, opportunistic approach to recording, describing, and analyzing brief moments of everyday technocultural activity. It has proved a useful term, referring on the one hand to the small-scale and shortduration nature of the events I am concerned with, and their transitory and momentary nature. On the other hand, the term alludes to the technological construction of this ethnographic research. The microethnography is brought into being by the act of video recording and its various actors (camera, tripod, PC, etc.), all of which are integral part(icipant)s of the event under scrutiny and the scrutiny as event. The presence of the researcher and the research technologies are inseparable from the networks under study. They too are (made of) part(icipant)s. The part(icipant)s of central concern to this study however, are the computer hardware, software, and human bodies, their parts, and their coming together in the intense and intimate circuits of gameplay.

A microethnographic approach suggests methodological strategies, both for analyzing gameplay and for identifying and conceptualizing relationships between technology agency and aesthetics in everyday technoculture across and between the virtual and the actual. It draws on conceptual and empirical work from cybercultural studies and science and technology studies. These fields of study are concerned with, on the one hand, the questioning of secure distinctions between people, culture, technologies, and the material world; and on the other with the tracing of the transmission or translation of agency among human and nonhuman entities. As such, a microethnography approach has to address and challenge the anthropocentrism of ethnography and anthropology to attend to nonhuman as well as human agencies in play.

\section{Event}

The object of study for a microethnography of videogame play then is not a media-cultural practice, a human subject, or a set of technologies, but rather the event in which the three come together (with the human and nonhuman researchers). Or, more accurately, it is the event that is constituted by, and constitutes, these part(icipant)s. Moreover, the event foregrounds the temporal dimension of videogame play, emphasizes the dynamic between the elements in play: entities coming together, material and aesthetic chains of cause and effect or feedback. Dan Fleming's (1996) consideration of the effects of diverse contexts for (in this case) toys in play is relevant here:

The effects we are going to be interested in are simultaneously in the formation of an object and in that object's consequences within the processes that formed it. In a way, therefore, itmight be better to talk about "events" rather than "objects." (pp. 10-11)

This resonates with actor-network theory's insistence on, on the one hand, the indivisibility of human and nonhuman agents and forces in any technosocial network or event, and on the other, the importance of the network over individual objects or subjects. To take the event as the primary object of study, then, is to look for the relationships constituted by, and constituting, hybrid and heterogeneous agents, rather than making assumptions about the primacy of either human or technological actors. As Bruno Latour (1992) puts it,

The distinctions between humans and non-humans, embodied or disembodied skills, impersonation or "machination", are less interesting than the complete chain along which competences and actions are distributed. (p. 243) 
Actor-network theory demonstrates that taking the relationships and networks that constitute objects and subjects as the focus of enquiry, rather than the objects and subjects themselves, is applicable to all technocultural phenomena, large and small, synchronic and diachronic. Whereas I would argue that an emphasis on the eventual could inform the study of all technocultural phenomena (not only those involving sophisticated technologies), it is particularly useful in the description and analysis of videogame play. Gameplay is a vivid example of the generation of new relationships and distributions of effect, affect, and feedback in everyday digital culture, of technicities not reducible to human identity and competence; a phenomenon that can only be adequately addressed through acknowledgement of its bringing together of heterogeneous part(icipant)s.

To explain this more clearly, I will refer briefly to a video microethnographic study of the playing of, and around, the PC game Lego Racers 2 by my two sons (then aged 3 and 4 years; Giddings, 2003, 2007). The computer game offers both a race track and its surrounding island environment for exploration by virtual Lego cars and people. After enthusiastically exploring the virtual environment of the game's first level ("Sandy Bay"), the boys transposed its topography into actual Lego. Those features of the game that afforded them the most pleasure, mountains, cliffs, a beach and the sea, were either constructed with Lego or MegaBlocks or drawn on paper to be lain on the floor. Cars and drivers were assembled from actual Lego and the virtual race was noisily actualized in and around the new game world.
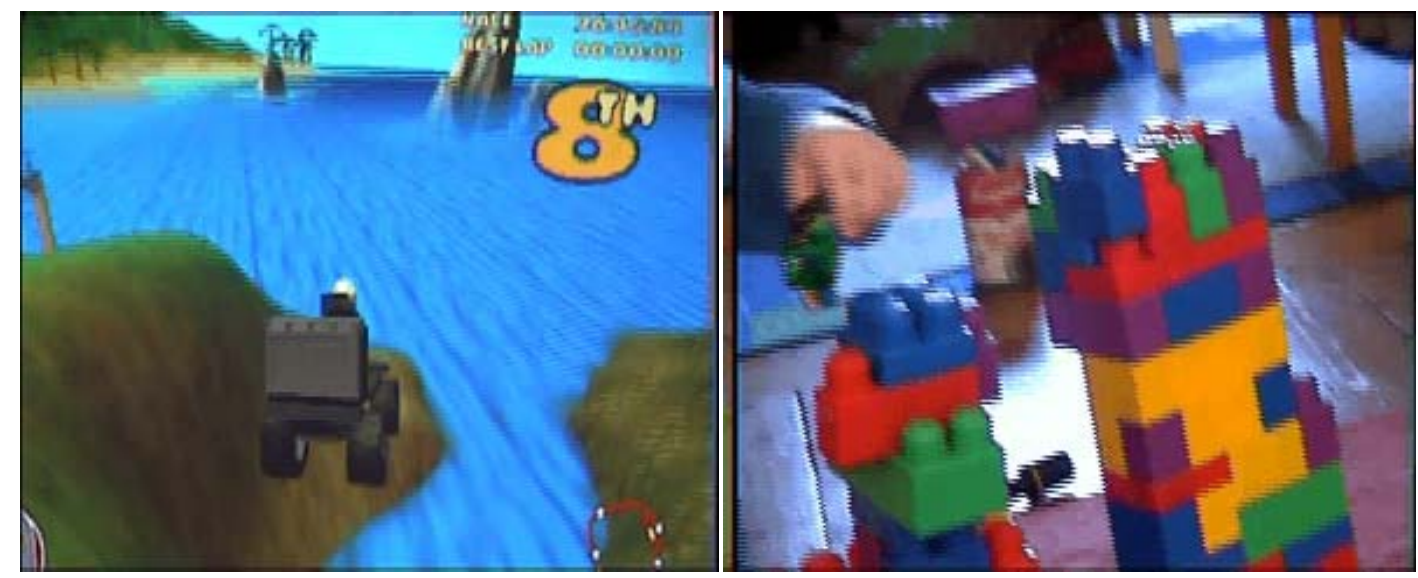

Children's play is often characterized by the translation or transposition of characters, topographies, and action from literature and media. In this event though, it became clear that it was not only the geographical features of Sandy Bay that were actualized (its mountains and coast) but also elements and forces arising from its existence as a ludic virtual world. This game world is coded to generate particular physical forces and behaviours, notably friction, acceleration and gravity, simulated forces designed to facilitate gameplay, and exploration rather than closely replicate actual world physics. There are resonances here with the mushroom story recounted earlier: In the Doone Valley, the power-up feature of videogames, and its (virtual) dynamic consequences, were transposed from game world to actual world. The emblem "mushroom" carried its affect and effect, from the virtual to the actual.

Videogame worlds are constituted by different part(icipant)s, different types of virtual phenomena, including objects and forces. The virtual space of Lego Racers 2 subjects its players to a range of simulated physical forces. Most notably, the boys quickly (and apparently unself-consciously), acclimatized themselves to the effects and possibilities of 
weakened virtual gravity. In balancing the forward momentum of the cars in response to the players' input with a lunar downward pull, this virtual physics became a ludic element in its own right, as evidenced by these boys' delight in driving their avatar cars up sheer cliffs and plunging them into the sea. And again, their translation of this virtual phenomenon-into actual movement and dynamics as they shift their plastic cars between the breakneck velocities of the living room circuit and the slow motion bounce and plummet over the brick mountains-highlights these virtual operations. A playful and vertiginous kinaesthesia is coded into the game world, affording new kinds of games. It exists between the operations of algorithms and variables and their engineering of embodied senses of control and resistance. In the study of this event, it became apparent that clear conceptual distinctions between virtual and actual space are unsustainable. Through play, these boys shifted across these two spaces with ease, their play adapting to the different environments, environmental resources, and the capabilities and possibilities they afforded. The virtual space in this event of gameplay does not transcend the everyday and embodied, it is a real space to be explored and in which the player can act, and be acted on. The virtual and the actual are both real, and in this event were each contained within the other, intertwining, each inflected by the other. Neither pre-exist the play-event itself though, rather they are reciprocally generated, produced in and through play events.1

Videogames are often celebrated as interactive media, as facilitating human activity and agency in media culture and on the screen. Yet it becomes clear here that videogame players are acted on as much as they act, that they must work out what the machine wants them to do (or what it will allow them to do) as well as engage with it imaginatively. A key term here is agency, or perhaps the less politically loaded behaviour. Neither agency nor behaviour can be restricted to the human participants here. Moreover, we are not looking at clear distinctions between human subjects and nonhuman objects, but the game event as one constituted by the playful translation of agency, the eccentric circuits of effect and affect, between human and nonhuman components.

\section{Microethology}

The term ethnography - the writing of people-itself establishes the human as the key object of research. As such, it is not adequate to the study of events constituted by human and nonhuman actors. 2 In its place I adopt and adapt ethology. The term ethology originates in the study of animal behaviour, the affects and capacities of animals and their environment. Gilles Deleuze (1992), with his rejection of ontological distinctions between animal and human bodies (and parts of bodies) as well as between the artificial and the natural, extends ethology to studies,

Which define bodies, animals or humans by the affects they are capable of ... Ethology is first of all the study of the relations of speed and slowness, of the capacities for affecting and being affected that characterize each thing. For each thing these relations and capacities have an amplitude, thresholds (maximum and minimum) and variations or transformations that are peculiar to them. And they select, in the world or in Nature, that which corresponds to the thing; that is, they select what affects or is affected by the thing, what moves or is moved by it ... an animal, a thing, is never separable from its relations with the world... The speed or slowness of metabolisms, perceptions, actions and reactions link together to constitute a particular individual in the world. (pp. 627-628)

It should be noted that Deleuze's (1992) use of the term affect is not limited to the experiential and emotional aspects of human embodiment and subjectivity, although these 
latter would be included within it, or, rather, constituted as particular products of it. "Bodies" for Deleuze (following Spinoza) are also animals, microscopic particles, organs, machines, a body of literature, and chemicals. As the quote above indicates, bodies are defined in part by their affect on the bodies around and within them and by the affect these other bodies have on them. Ethology's concern then is with behaviour.

Animals are defined less by the abstract notions of genus and species than by a capacity for being affected, by the affections of which they are "capable" by the excitations to which they react within the limits of their capability. (Deleuze, 1988, p. 27)

If the humanist etymological roots of the term ethnography are shed along with its anthropocentric practices, then what the study of gameplay needs is a microethology, a study and description of the behaviours, affects, and mutual becomings of a microworld or the micronature of part(icipant)s, of fingers and thumbs, mushrooms and data projectors, algorithms and aptitude, playing bodies both human and nonhuman, rather than the a priori establishment of human, machinic, or textual bodies as the objects of study.

\section{Beyond Identification}

Conventionally, in film and media studies, any event of screen media engagement is primarily read in terms of psychic identification by a human subject with representations of human subjects on the screen (e.g., Metz, 1985). In the small studies under consideration here, such assumptions are undermined. In videogames, on one level, the shifting of players' "identification" with screen images is driven more by the demands of the various games in play than any sense of ideological investment by a subject positioned in relation to a fictional protagonist.

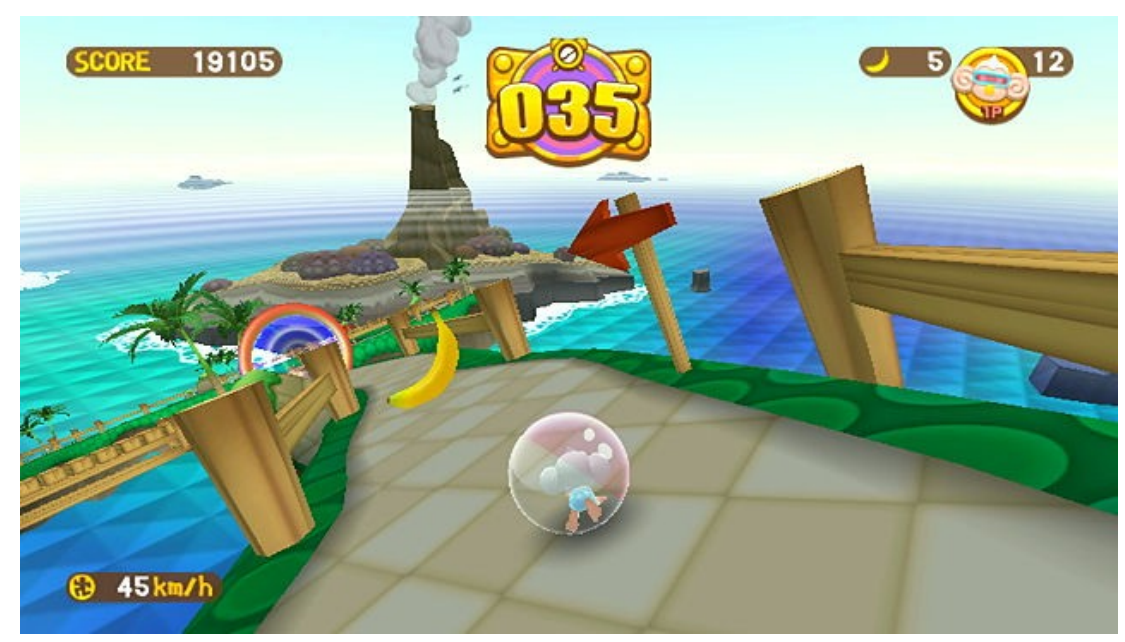

Other agencies are imaginatively displaced onto other nonhuman part(icipant)s in the shift from play in virtual to actual worlds. In Super Monkey Ball for example, although the player chooses a monkey character according to personal taste (all are extremely cute), to undertake the adventure for them, the monkeys are not avatars. The game dynamic requires the player to control the monkey's environment: it is the virtual landscape/architecture or platform that is tipped and tilted, the monkey (in its ball) that rolls forward through the level. The game mechanic is based in conflict between the player and the very element of the game immediately engaged with by the player. The platform, whether narrow bridge or tricky jump, would seem to be at once the avatar (the game-element under the player's control) and the enemy. Theories of identification would look to the player's subjective 
investment in the anthropomorphic monkey in his or her battles with his or her environment. Yet in the game world, the player's agency is exercised through the environment: the player and platform are locked together in a feedback loop to propel the monkey (motivated only by virtual gravity) to its goal.

Another microethnographic study illustrates how identification with (or, at least, pleasure in playing with) screen media characters becomes, in videogame play, only one affectional relationship among many (Giddings \& Kennedy, 2008). In this study, two players (the researchers themselves) cooperatively learn to play the videogame Lego Star Wars. The research aim was to identify, describe, and theorize the various agents at play, particularly the game world and its nonplayer character denizens and their effects and affects on the human players. One moment in this play-event illustrates something of the application, and limitation, of the notion of identification in videogame play. It also indicates something of the frustrations and excitement of constituting a new game world:

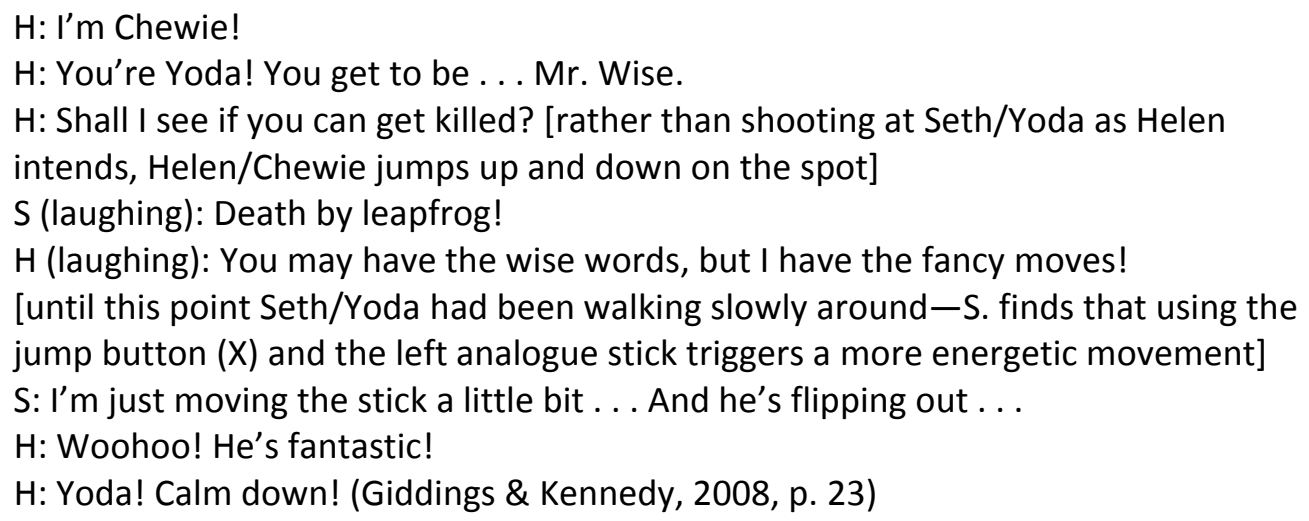

Here then, the initial pleasure of the players in recognizing their avatars from their knowledge of the cinematic world of Star Wars quickly shifts to an exploration of the material affordances that these avatars possess within the game world as elements of that software world. Aesthetic pleasures familiar from cinema and television do not disappear; rather they become one pleasure in a set, some of which are unique to digital media. Or rather, they enter into circuits of effect and affect with these newer pleasures. Hence, the players' hilarity at the Yoda avatar's "flipping out" is driven by the incongruity of the meditative, sage-like film character exploding into spectacular cybernetic action at the slightest nudge of an analogue stick.

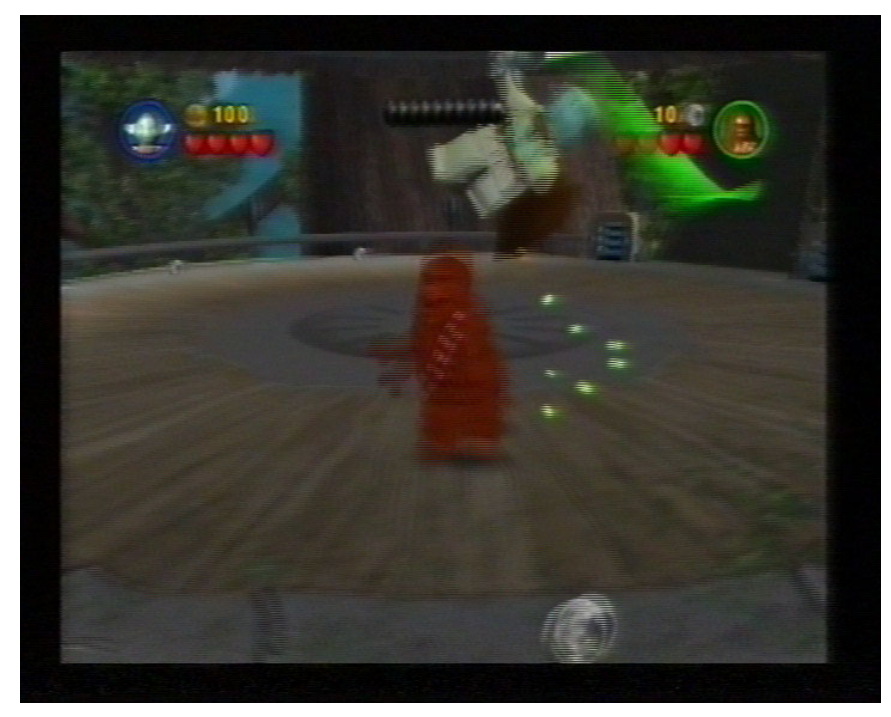


An analytical focus on avatars and nonplayer character denizens runs the risk of reinforcing anthropocentrism however. It should be noted that much of the progress toward the successful instantiation of the Lego Star Wars game event was achieved by one set of actual components (eyes, brains, thumbs, controllers, buttons, etc.) responding to the suggestions or demands of virtual components. Grates, levers, vents, and doors glow as avatars approach them, willing the use of the Force (the circle button on the PlayStation 2 controller) to reveal secrets or treasure, or directing thumbs to nudge sticks that in turn propel avatars in the directions of actions that the game-as-ludic-software-system as a whole prefers or demands. That human part(icipant)s are also composed of parts that come into play with the parts of the game system is evident in the numerous occasions in which the player's brain attempts to fire a soft weapon, but his or her thumb insists on making the avatar jump, whereas his or her eyes may well-be tracking a character on screen that is not actually their avatar.

As has already been observed, to conduct a microethology implies a concern with the material operations of affect as well as (or before) any concern with psychic engagement with avatars or other discrete game elements. As the term identification indicates, notions of (human) identity and subjectivity prove very resilient. Studies of videogame play and players to date nearly always reproduce a priori assumptions of the primacy of human agency and identity. These assumptions should be questioned on (at least) two counts.

First, the playful and aesthetic relationships between the human and the nonhuman that constitute the videogame play event are not only psychic, imaginary, significatory, or textual. They are also material: mechanical and cybernetic circuits of embodied feedback across the virtual and the actual. Second, despite postmodernist and poststructuralist claims to its multiplicity or its decentring, the coherent human subject remains a foundational object of knowledge in cultural theory. Although the part(icipant)s in and of Aarseth's (1997) game/system are by and large nonhuman, their refusal to be amalgamated into a coherent object or whole ("the game" or "a computer") resonates with recent work in science studies and media studies that effects analogous operations on the figure of the coherent human subject. Donna Haraway's (2004) work in this field is key. She describes object/bodies that do not preexist but whose boundaries "materialize in social interaction among humans and nonhumans', material-semiotic actors that include 'the machines and other instruments that mediate exchanges at crucial interfaces and that function as delegates for other actors' functions and purposes" (p. 68). Matthew Fuller (2005), in his study of the technocultural ecology of pirate radio, paraphrases Nietzsche thus,

The "subject" is merely a regent at the head of a "communality" of processes all of which are in a continual state of agitation, in and outside of any named body, with relations between them fluctuating. The subject thus emerges from the sustained interactions of these "subordinate" forms ... The subject is a case of perspectival position rather than a categorical a priori condition of knowledge (p. 63).

In the Lego Racers event, the boys would seem to slip between a number of 'identifications": being the virtual Lego men and being the virtual Lego car-driver dyad or the car itself; being the constructors of these men, cars, and car-men; being at once the child playing with the actual Lego car-men and coextensive with the car or car-men they are propelling around the room; being the player of a videogame and being a meta-playerperhaps the computer (or game-system) itself - in an actual game. The naming of these hybrid entities is not a rhetorical or metaphorical move however: a microethological 
approach would look for the affected and affecting bodies (or part(icipant)s) in this event. Thus the players here are generated by the game event, compiled from parts that are human and nonhuman, virtual and actual.

\section{Conclusion: Collusion}

Microethnography/microethology bring to cybertextual analysis an attention to the operations of virtual circuits and components with, and as, their relationships to human players, hardware, and actual environments. A key challenge for a microethology of gameplay is to describe and analyze these material events as generated and constituted by various bodies and agents - part(icipant)s both human and nonhuman, hard and softwithout reinscribing humanist a priori distinctions between subject and object. Assumptions or assertions of subject / object distinctions in gameplay, at best allow attention to only some of the gameplay event's components: the screen images but not the human player's behaviours; physical movements but not rule sets, and so on. But at worse, such distinctions deny the co-constitutional nature of gameplay as intense, intimate, and cybernetic-as relations and transformations of speed, slowness, and affect between all part(icipant)s: they break the circuit.

What might the starting point, the focus, be of a microethology of gameplay? In place of "identification" as the privileged term for the relationship of human and nonhuman in videogame play, I suggest collusion. The word has a ludic etymology (co-lusion), so to collude is not only "to work with another" or "act in concert with", but also "to act in play", to come together in, and as, play. The word does not in itself assume anything about the nature of the colluding entities, but indicates the videogame's material distribution of agencies and the positioning of agents, bodies, or part(icipant)s. The event is constituted by the coming together in play, the collusion of material and imaginary elements: the operations of games (their conventions, rules, and prescriptions), embodied knowledge and technicities (and pleasures, anxieties, frustrations, imagination), play practices (role play, toy play), screen media images and characters, virtual game worlds (and their physics, automata, and affordances), and all sorts of bodies.

Questioning the conceptual centrality of the human subject does not mean that human desires, anxieties, identifications, and investments are not in play in media technoculture. In the events briefly described above, the different games are spun into being, through the tastes, personalities, and abilities - the technicities - of the human part(icipant)s as well as the material affordances of computer hardware and software simulacra, and beyond-into whatever resources are at hand for play, including actual toadstools and fields. If code and information must be understood as real, material, of the world, then so too can the intangible yet real, embodied yet distributed, monstrous, operations of human partsperception, imagination, creativity, anxiety, play-without always already reducing these to the reassuring synechdoches of "identity" and "subjectivity." 


\section{Notes}

1. See Giddings (2007) for a fuller account of this study

2. Indeed, following Latour (1992), there is no instance of culture or social life constituted only by human actors: The cultural is always technocultural.

\section{References}

Aarseth, E. (1997). Cybertext: Perspectives on ergodic literature. Baltimore: Johns Hopkins University Press.

Bolter, J. D., \& Grusin, R. (1999). Remediation: Understanding new media. Cambridge: MIT Press.

Deleuze, G. (1988). Spinoza: practical philosophy. San Francisco: City Lights Books.

Deleuze, G. (1992). Ethology: Spinoza and us. In J. Crary \& S. Kwinter (Eds.), Zone 6: Incorporations (pp. 625-633). New York: Zone Books.

Fleming, D. (1996). Powerplay: toys as popular culture. Manchester, UK: Manchester University Press.

Fuller, M. (2005). Media ecologies: materialist energies in art and technoculture. Cambridge: MIT Press.

Giddings, S. (2003). Circuits: A video essay on virtual and actual play. In M. Copier \& J. Raessens (Eds.), Level up: Digital games research conference [CD-ROM]. Utrecht, The Netherlands: Faculty of Arts, Utrecht University Press.

Giddings, S. (2007). I'm the one who makes the Lego Racers go! Studying virtual and actual play. In S. Dixon \& S. Weber (Eds.), Growing up online: Young people and digital technologies (pp. 35-49). New York: Palgrave Macmillan.

Giddings, S., \& Kennedy. (2008). Little Jesuses and fuck-off robots: on aesthetics, cybernetics and not being very good at Lego Star Wars. In M. Swalwell \& J. Wilson (Eds.), The pleasures of computer gaming: Essays on cultural history, theory, and aesthetics (pp. 13-33). Jefferson, NC: McFarland.

Haraway, D. (1990). A manifesto for cyborgs: Science, technology, and socialist feminism in the 1980s. In L. J. Nicholson (Ed.), Feminism/postmodernism (pp. 190-234). New York:

Routledge.

Haraway, D. (2004). The Haraway reader. London: Routledge.

Latour, B. (1992). Where are the missing masses? The sociology of a few mundane artefacts. In W. Bijker \& J. Law (Eds.), Shaping technology/building society: Studies in sociotechnical change (pp. 225-258). Cambridge: MIT Press.

Lister, M., Dovey, J., Giddings, S., Kelly, K., \& Grant, I. (2003). New media: A critical introduction. London: Routledge.

Manovich, L. (2001). The language of new media. Cambridge: MIT Press.

Metz, C. (1985). Psychoanalysis and cinema: The imaginary signifier. London: Macmillan.

Salen, K., \& Zimmerman, E. (2003). The rules of play: Game design fundamentals. ambridge: MIT Press. 